\title{
16. Zeit gegen Geld? Der Austausch von Unterstützung zwischen den Generationen
}

\author{
Daniela Klaus \& Katharina Mahne
}

\section{Kernaussagen}

Intergenerationale Transfers folgen 2014 demselben Muster wie 1996: Innerhalb einer Familie fließen materielle Ressourcen häufiger von den älteren zu den jüngeren Generationen und instrumentelle Hilfen häufiger in die andere Richtung.

Materielle Transfers an jüngere Generationen finden im Jahr 2014 häufiger statt als im Jahr 1996: Die 40- bis 54-jährigen Eltern unterstützen im Jahr 2014 zu 44,9 Prozent ihre erwachsenen Kinder mit Geld- und Sachgeschenken. Im Jahr 1996 waren es mit 35,6 Prozent weniger. Auch bei den 55bis 69-Jährigen sind materielle Transfers an erwachsene Kinder im Jahr 2014 häufiger (37,5 Prozent) als noch vor 18 Jahren (29,5 Prozent).

Die Häufigkeit materieller Transfers von Großeltern an Enkelkinder hat sich zwischen 1996 und 2014 fast verdoppelt: 8,1 Prozent der Großeltern im Alter von 55 bis 69 Jahren haben im Jahr 1996 materielle Transfers an ihre Enkelkinder gegeben, 2014 sind es mit 15,8 Prozent fast doppelt so viele. Auch bei den 70- bis 85 -jährigen Großeltern haben sich die Transferraten im selben Zeitraum von 14,8 Prozent auf 28,4 Prozent fast verdoppelt.

Im Jahr 2014 erhalten ältere Familiengenerationen seltener instrumentelle Hilfen von jüngeren als im Jahr 1996: Während im Jahr 1996 noch etwa ein Fünftel (19,5 Prozent) der ältesten Eltern (70 bis 85 Jahre) Hilfe im Alltag von den Kindern bekam, ist es im Jahr 2014 nur noch gut ein Zehntel (11,7 Prozent). Auch von den Enkelkindern erhalten die 70- bis 85-Jährigen im Jahr 2014 seltener instrumentelle Hilfe (1996: 6,5 Prozent; 2014: 2,6 Prozent).

\subsection{Einleitung}

Es gibt eine breite und umfangreiche Forschung zur Solidarität zwischen den Generationen. Ein Thema, das auch seit Jahren den öffentlichen und politischen Diskurs mitbestimmt, indem anhaltend und kontrovers über einen möglichen Rückgang des Zusammenhalts zwischen den Generationen diskutiert wird (vgl. Kapitel 14). Dabei konnte bereits Ende der 1990er Jahre gezeigt werden, dass zwischen den Generationen beträchtliche Solidarleistungen füreinander erbracht werden (Kohli, Künemund, MotelKlingebiel, \& Szydlik 2005; Kohli \& Künemund
2001). Hierbei zeichnet sich ein Muster ab, für das die Bezeichnung, reziprokes Kaskadenmodell (Marbach 1997) geprägt wurde und das auch für spätere Zeitpunkte repliziert werden konnte (Mahne \& Motel-Klingebiel 2010). Demnach fließen finanzielle Transfers innerhalb der Familie hauptsächlich von den älteren $\mathrm{zu}$ den jüngeren Generationen: Erwachsene Kinder und Enkelkinder erhalten häufiger materielle Zuwendungen von ihren Eltern und Großeltern als sie umgekehrt an diese geben. Parallel dazu werden instrumentelle Hilfen vornehmlich in 
Richtung der Generationen aufwärts geleistet: So erhalten Eltern und Großeltern vor allem in späteren Lebensphasen mehr praktische Unterstützung im Alltag und in Notfällen als sie an nachfolgende Generationen geben.

Das reziproke Kaskadenmodell wird als positive Folge des wohlfahrtsstaatlichen Ausbaus interpretiert. Erst der öffentliche Generationenvertrag, der vor allem die Grundlage für das umlagebasierte Rentensystem ist, ermöglicht die innerfamiliale und zugleich sehr flexible Umverteilung monetärer Ressourcen. So werden erwachsene Kinder gezielt je nach Bedarf zum Beispiel bei Arbeitslosigkeit oder Scheidung von ihren Eltern unterstützt (Motel-Klingebiel, Mahne, \& Huxhold 2010). Gleichzeitig versetzt die prinzipielle finanzielle Unabhängigkeit voneinander die Generationen in die Lage, sich auf instrumentelle und auch emotionale Unterstützung $\mathrm{zu}$ konzentrieren (Künemund \& Rein 1999). Europaweite Befunde belegen, dass erwachsene Kinder umso häufiger von ihren Eltern unterstützt werden, je universeller und großzügiger die wohlfahrtsstaatlichen Leistungen ausfallen (Hank 2015; Deindl, Brandt, \& Hank 2014). Befürchtungen, dass umfangreiche wohlfahrtsstaatliche Unterstützung an Familien die intergenerationale Solidarität gefährden könnte (,crowding out'), sind also unbegründet. Im Gegenteil: ein starker Wohlfahrtssaat scheint sie sogar eher zu stimulieren (Motel-Klingebiel, Tesch-Römer, \& von Kondratowitz 2005). Im europäischen Vergleich bewegen sich intergenerationale Transfers in Deutschland auf einem mittlerem Niveau (Albertini \& Kohli 2013; Alessie, Angelini, \& Pasini 2014).

Gesellschaftliche Trends der letzten Jahre geben Anlass zu der Vermutung, dass es zu Verschiebungen der bekannten Transfermuster zwischen den Generationen gekommen sein könnte. Längere Ausbildungszeiten, brüchigere Erwerbsbiografien, neue Herausforderungen bezüglich der Vereinbarkeit von Beruf und $\mathrm{Fa}$ milie, gestiegene Mobilitäts- und Flexibilitätserfordernisse sowie rückläufige (Enkel-)Kinderzahlen könnten dazu beigetragen haben, dass das Unterstützungspotenzial, das jüngere Familienmitglieder für Ältere darstellen, zurückgegangen ist. So stehen Jüngeren heute möglicher- weise weniger Ressourcen zur Verfügung, um ihre Eltern und Großeltern zu unterstützen. Das dürfte sowohl auf monetäre als auch auf Zeitressourcen zutreffen. Mehr noch: Es ist zu vermuten, dass junge Generationen heute noch länger von finanziellen Zuwendungen ihrer Eltern abhängig sind, weil ihr beruflicher Einstieg und ihre Etablierung auf dem Arbeitsmarkt zunehmend in höherem Alter erfolgen. Ob und inwiefern die finanzielle Ausstattung in der Elterngeneration solcherlei Unterstützung erlaubt, ist zu prüfen. Es ist jedoch wahrscheinlich, da sich ihre wirtschaftliche Situation im Durchschnitt in den letzten Jahren verbessert hat (vgl. Kapitel 6 und 7; Vogel \& Künemund 2014). Neben einer verbesserten materiellen Lage hat die gestiegene gemeinsame Lebenszeit von familialen Generationen (vgl. Kapitel 14) zum Beispiel die Möglichkeit geschaffen, dass Großeltern ihre finanzielle Unterstützung auch direkt an erwachsene Enkelkinder richten können - beispielsweise indem sie sich an deren Ausbildungskosten beteiligen oder im Bedarfsfall wie Arbeitslosigkeit finanziell aushelfen. Ein europäischer Vergleich zeigt für Deutschland die höchsten Anteile großelterlicher finanzieller Unterstützung (Igel 2012). Anzunehmen ist weiterhin, dass die verbesserte Gesundheit der Älteren intergenerationale Hilfe und Unterstützung im Alltag seltener notwendig werden lassen. Möglicherweise rückgängige Transferquoten müssen hier also nicht zwangsläufig eine schlechte Nachricht sein. Außerdem können Unterstützungsbedarfe der älteren Familiengenerationen häufiger durch alternative, professionelle Dienstleistungsangebote im Wohnumfeld (vgl. Kapitel 20) oder durch zunehmend an Bedeutung gewinnende nicht-familiale Beziehungen (vgl. Kapitel 17 und 21) befriedigt werden, um auf diese Weise zur Erleichterung der alltäglichen Lebensführung beizutragen - gerade auch wenn Familienmitglieder diese Hilfe nicht leisten können.

Besondere Aufmerksamkeit bei der Untersuchung des Wandels intergenerationaler Transfers ist auf die sich über den Lebenslauf hinweg verändernde Position der Menschen im Generationengefüge $\mathrm{zu}$ richten. Auswertungen auf Basis des Deutschen Alterssurveys (DEAS) von 2008 haben gezeigt, dass die 40 - bis 54 -Jährigen 
am stärksten in das Transfergeschehen eingebunden sind: Sie geben häufig Unterstützung an Eltern, Kinder und Großeltern, werden aber auch umfangreich von ihnen unterstützt (Mahne \& Motel-Klingebiel 2010). Aus der vorliegenden Forschung ist zudem bekannt, dass sich analog $\mathrm{zu}$ sich verändernden Ressourcen und Bedarfen der verschiedenen familialen Generationen über den Lebenslauf hinweg eine Verschiebung von Gabe hin zu mehr Erhalt zeigt. Offenbar ist der intergenerationale Austausch von Personen mittleren Erwachsenenalters am ehesten zwischen Geben und Nehmen ausgeglichen. $A b$ dem 50. Lebensjahr beginnt sich die Austauschbilanz älterer Personen hin zu Netto-Gebern zu verschieben. Erst im hohen Alter kehrt sie sich um: Personen ab dem 80. Lebensjahr erhalten im Allgemeinen mehr Unterstützung von jüngeren Generationen als sie umgekehrt geben (Litwin, Vogel, Künemund, \& Kohli 2008; Vogel 2008). Bedingt durch eine spätere Familiengründung und die Entwicklung, dass Menschen nicht nur länger leben, sondern auch länger gesund, unabhängig und mobil bleiben (vgl. Kapitel 8), ist anzunehmen, dass sich Phasen intensiven Austausches in höhere Altersgruppen verlagert haben oder sich generell ausgedehnt haben.

Neben veränderten Ressourcen und Bedarfen der beteiligten familialen Generationen, die das Transfergeschehen maßgeblich beeinflussen, können sich die Transfermuster für Män-

\subsection{Datengrundlage ${ }^{1}$}

Daten. Zur Untersuchung eines möglichen Wandels im intergenerationalen Transfergeschehen stehen Daten für alle vier Ersterhebungen $(1996,2002,2008,2014)$ zur Verfügung. In der folgenden Ergebnisdarstellung werden lediglich die erste (1996) und die derzeit letzte DEAS-Erhebung (2014) einander vergleichend gegenübergestellt. Ergebnisse zu den Zwischen-

1 Die Daten des DEAS können für wissenschaftliche Zwecke kostenlos beim Forschungsdatenzentrum des DZA (www.fdz-dza.de) bezogen werden. ner und Frauen unterschiedlich darstellen oder verändern. Die Befundlage hierzu ist allerdings nicht eindeutig (Kahn, McGill, \& Bianchi 2011). Zwar gelten Frauen weithin als die ,kinkeeper der Familie (Rosenthal 1985) und aktuelle Studien zeigen, dass Frauen häufiger helfen als Männer und dabei vor allem zeitintensive Aufgaben übernehmen (vgl. Kapitel 12; Alessie et al. 2014; Deindl et al. 2014). Allerdings ist aufgrund der Zunahme der beruflichen Einbindung von Frauen - und damit geringeren Zeitressourcen - eine rückläufige Quote der von ihnen erbrachten instrumentellen Transfers über die Zeit hinweg zu vermuten. Parallel dazu wäre aufgrund gestiegener eigener Einkommen ein Anstieg der Gabe materieller Transfers denkbar.

Im vorliegenden Kapitel werden die folgenden Fragen untersucht:

1. Haben sich die Prävalenzraten intergenerationaler Unterstützung verändert?

2. Folgt der intergenerationale Austausch weiterhin einem reziproken Kaskadenmodell?

3. Zeichnet sich eine Zunahme des direkten Austausches zwischen Großeltern und Enkelkindern $\mathrm{ab}$ ?

4. Zeigen sich Unterschiede zwischen den Geschlechtern?

jahren finden sich im Tabellenanhang. Einbezogen werden alle Erstbefragten im Alter zwischen 40 und 85 Jahren.

Transferraten. Betrachtet werden materielle und instrumentelle Transfers. Ausgehend von den Befragten werden Transfers von und für vier Familiengenerationen berücksichtigt: Eltern, Kinder, Enkelkinder und Großeltern. In jeweils beide Tauschrichtungen wird erfasst, ob in den letzten zwölf Monaten vor der Befragung entsprechende Transfers geflossen sind. Materielle Unterstützung wird darüber abgebildet, ob ande- 
ren größere Geld- oder Sachgeschenke gemacht oder regelmäßige finanzielle Unterstützung gewährt wurde und ob umgekehrt derartige Leistungen erhalten wurden. Instrumentelle Unterstützung wird über Hilfe im Haushalt abgebildet, die geleistet oder erhalten wurde. Entsprechend liegen für jede der beiden Unterstützungsarten acht dichotome Indikatoren vor, die mit (0) ,nein und (1) ,ja kodiert sind. Gültige Werte liegen nur dann vor, wenn mindestens ein Mitglied der betrachteten Generation lebt. Transfers von und an Kinder wurden nur für Kinder berücksichtigt, die mindestens das 18. Lebensjahr erreicht haben und in einem eigenen Haushalt leben (Mahne \& Motel-Klingebiel, 2010). Auf diese Weise soll haushaltsinterne Hilfe innerhalb einer Bedarfsgemeinschaft ebenso ausgeschlossen werden wie die Unterstützung noch minderjähriger Kinder.
Stief-, Adoptiv- und Pflegekinder sind leiblichen Kindern gleichgestellt.

Gruppierungsvariablen. Zur Prüfung von Altersunterschieden wurden - analog zur Schichtung der Stichprobe - drei Altersgruppen verwendet: 40-54 Jahre, 55-69 Jahre und 70-85 Jahre. Zudem wurden Geschlechterunterschiede untersucht.

Analyse. In den Abbildungen und im Text sind gewichtete (gruppenspezifische) prozentuale Anteile angegeben. Um Unterschiede zwischen 1996 und 2014 auf Signifikanz zu testen, wurden logistische Regressionen berechnet, wobei für die Stratifizierungsvariablen Altersgruppe, Geschlecht und Region (Ost-/Westdeutschland) kontrolliert wurde. Das genaue Vorgehen ist in Kapitel 2 beschrieben.

\subsection{Ergebnisse}

In Abbildung 16-1 sind die intergenerationalen Transferflüsse von Geld- und Sachleistungen sowie instrumenteller Hilfe für die einzelnen Generationenbeziehungen dargestellt - separat für die drei Altersgruppen und jeweils vergleichend für die Jahre 1996 und 2014. Die Anordnung der Generationen spiegelt deren Abfolge wider, wobei jeweils die mittlere Generation unterschiedlichen Alters - die auskunftsgebende Befragungsperson stellt. Für jede der von ihr aus betrachteten Generationen sind die Anteile derer angegeben, die mindestens eine lebende Person der jeweiligen Generation haben beziehungsweise bezogen auf die Generation der Kinder: mindestens ein erwachsenes Kind, das nicht mehr im elterlichen Haushalt wohnt. Die Fläche der generationenaufwärts oder -abwärts gerichteten Balken bildet den Umfang der Transfers in die jeweilige Richtung ab. Sind keine Balken eingezeichnet, so wurde in der jeweils betrachteten Beziehung und Richtung kein Transfer berichtet. Werden in den Abbildungen keine Transferraten berichtet, so verweist das auf eine Gruppengröße von zehn oder weniger Fällen in der jeweiligen Konstellation und ent- sprechend wurde auf die Präsentation der Werte verzichtet.

\section{Materielle Transfers an jüngere Generationen finden im Jahr 2014 häufiger statt als im Jahr 1996.}

Insgesamt sind die intergenerationalen Transfers auf einem hohen Niveau verblieben. Es gibt keine Hinweise darauf, dass sie in ihrer Gesamthäufigkeit zurückgegangen wären. Bei genauerer Betrachtung konkreter Generationenbeziehungen und Transferrichtung werden allerdings zwei Trends sichtbar: Erstens geben Personen in der zweiten Lebenshälfte 2014 häufiger materielle Unterstützung als 1996 - vor allem an ihre Kinder und Enkelkinder, aber auch an ihre Eltern. Zweitens erhalten sie von ihren Kindern und Enkelinnen und Enkeln seltener instrumentelle Hilfe.

Das häufigere Auftreten materieller Transfers zeigt sich erwartungsgemäß lebensphasen- beziehungsweise familienphasenspezifisch: So sind in der Gruppe der 40- bis 54-Jährigen signifikant häufiger Geld- und Sachleistungen an eige- 
ne Eltern (1996: 3,8 Prozent, 2014: 6,8 Prozent) und erwachsene Kinder auszumachen (1996: 35,6 Prozent, 2014: 44,9 Prozent). In der mittleren Altersgruppe der 55- bis 69-Jährigen sind vor allem die materiellen Transfers an erwachsene Kinder (1996: 29,5 Prozent, 2014: 37,5 Prozent) und Enkelkinder häufiger (1996: 8,1 Prozent, 2014: 15,8 Prozent). In der ältesten Gruppe (70 bis 85 Jahre) sind ausschließlich die Anteile von Personen größer, die Geld- und Sachgeschenke an ihre Enkelkinder geben (1996: 14,8 Prozent, 2014: 28,4 Prozent). Dabei ist es offenbar kurzfristig zu dem Anstieg materieller Transfers für erwachsene Kinder gekommen, denn zwischen 1996 und 2008 ist diese Unterstützung tendenziell zurückgegangen (vgl. Tabelle A 16-1 im Anhang, Mahne \& Motel-Klingebiel, 2010). Die deutlich häufigere materielle Unterstützung von außerhalb des elterlichen Haushaltes wohnenden Kindern deutet möglicherweise auf deren gestiegene finanzielle Abhängigkeit von ihren Eltern hin. So scheinen materielle Zuwendungen im Jahr 2014 seltener auf die Zeit der Ausbildung und des Berufseinstieges der jungen erwachsenen Kinder beschränkt zu bleiben, sondern schließen offenbar auch die Phase der Familiengründung oder auch Erwerbsunterbrechungen im Erwachsenenalter ein. Der insgesamt beträchtliche Anstieg des Auftretens (groß-)elterlicher Zuschüsse impliziert neben dem gestiegenen Unterstützungsbedarf seitens der jüngeren Generationen, dass die älteren Generationen als Geber hierzu finanziell in der Lage sind - und zwar nicht nur, solange sie selbst erwerbstätig sind, sondern auch dann, wenn sie das Rentenalter erreicht haben. Dahinter ist eine Verbesserung der finanziellen Ressourcenausstattung in allen Altersgruppen der zweiten Lebenshälfte zu vermuten (vgl. Kapitel 6 und 7).

\section{Im Jahr 2014 erhalten ältere Familiengenerationen seltener instrumentelle Hilfen von Jüngeren als im Jahr 1996.}

Signifikante Rückgänge für den Erhalt instrumenteller Hilfe zeigen sich zwischen 1996 und 2014 nur für die mittlere und die älteste Altersgruppe und damit besonders für diejenigen Personen, die potenziell verstärkt auf Hilfe bei der Alltagsführung angewiesen sein dürften. Besonders deutlich wird diese Entwicklung in der Gruppe der 70- bis 85-Jährigen: Hier ist der Anteil von Personen, die Unterstützung im Alltag von ihren erwachsenen Kindern erhalten, im Jahr 2014 (11,7 Prozent) geringer als im Jahr 1996 (19,5 Prozent). Auch von den Enkeln werden sie 2014 seltener durch Hilfe im Alltag unterstützt (1996: 6,5 Prozent, 2014: 2,6 Prozent). Hinter dieser Entwicklung werden zum einen auf Seiten der jüngeren Generation verschlechterte Bedingungen zur Leistung instrumenteller Hilfe vermutet: Vor allem dürften gestiegene Wohndistanzen zwischen den Generationen gerade das Erbringen praktischer Hilfe erschweren, da diese typischerweise eine gewisse räumliche Nähe voraussetzt (vgl. Kapitel 14). Zudem werden für (Groß-)Eltern zwischen 40 und 85 Jahren im Jahr 2014 geringere durchschnittliche Kinder- beziehungsweise Enkelzahlen beobachtet als im Jahr 1996 (vgl. Kapitel 15). Sind insgesamt weniger Kinder und Enkelkinder verfügbar, reduziert sich die Wahrscheinlichkeit, von einem dieser (Enkel-)Kinder Hilfeleistungen $\mathrm{zu}$ bekommen. Zum anderen ist auf Seiten der älteren Generation - hier als Empfänger - eine zunehmende Unabhängigkeit von den Nachkommen insofern zu vermuten, als gesundheitliche Einschränkungen insbesondere im hohen Alter seltener auftreten (vgl. Kapitel 8). Außerdem wird Unterstützung häufiger durch Personen aus dem Wohnumfeld wie Nachbarinnen und Nachbarn oder durch Freundinnen und Freunde geleistet. Schließlich macht es die gute materielle Lage der Älteren möglich, angesichts weiter gestreuter familialer Netze alternativ oder zusätzlich auch bezahlte Dienstleistungen in Anspruch zu nehmen. 
Abbildung 16-1: Transferraten intergenerationaler Unterstützung, nach Alter, in den Jahren 1996 und 2014 (in Prozent)

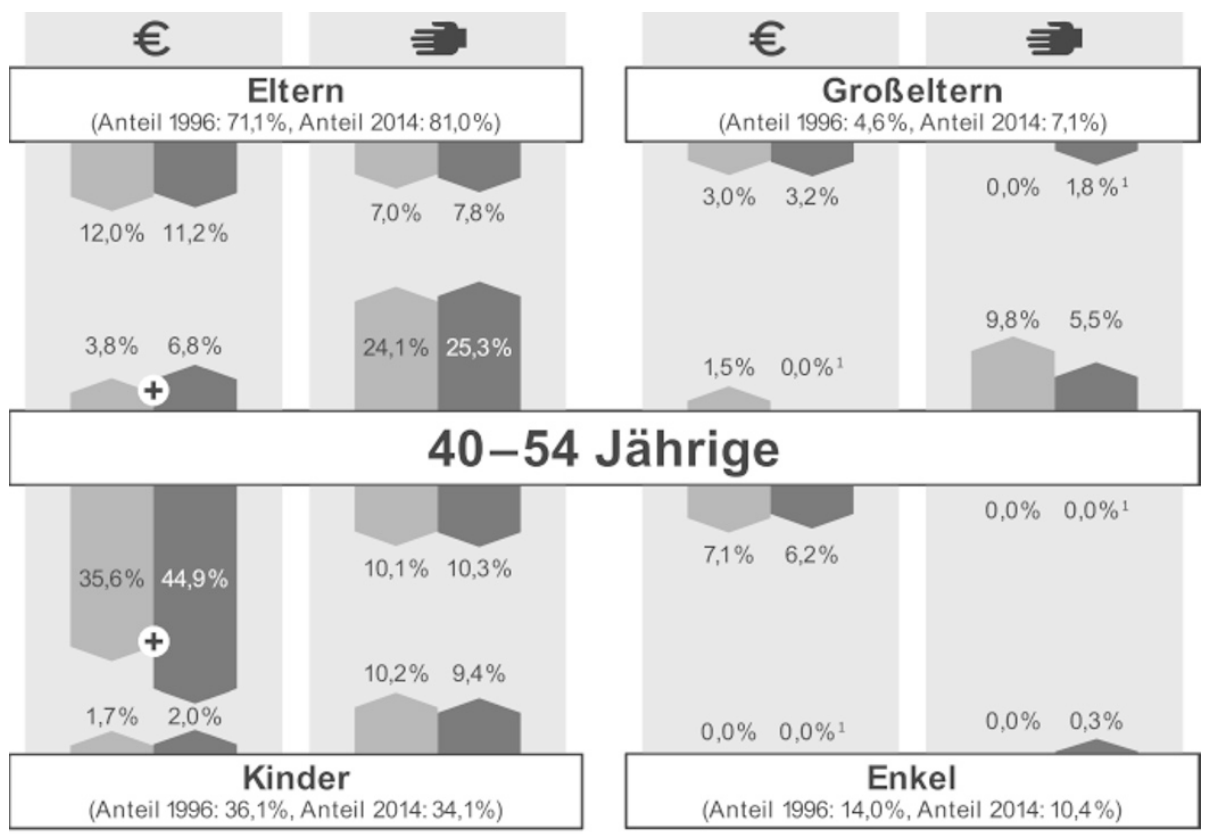

€ Materielle Transfers $\Rightarrow$ Instrumentelle Hilfe $\quad 1996 \quad \square 2014$

+ - Signifikante Veränderungen $(p<, 05)$ für den Zeitraum zwischen 1996 und 2014.

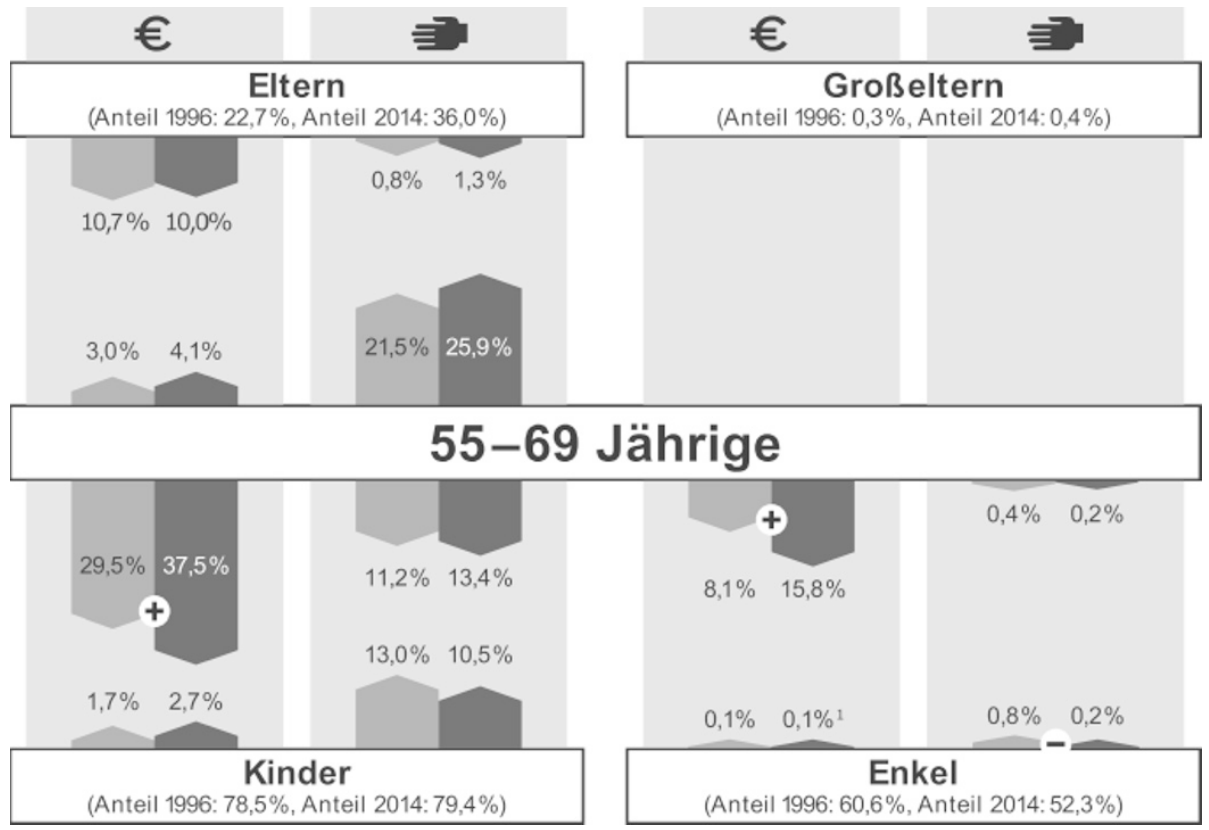




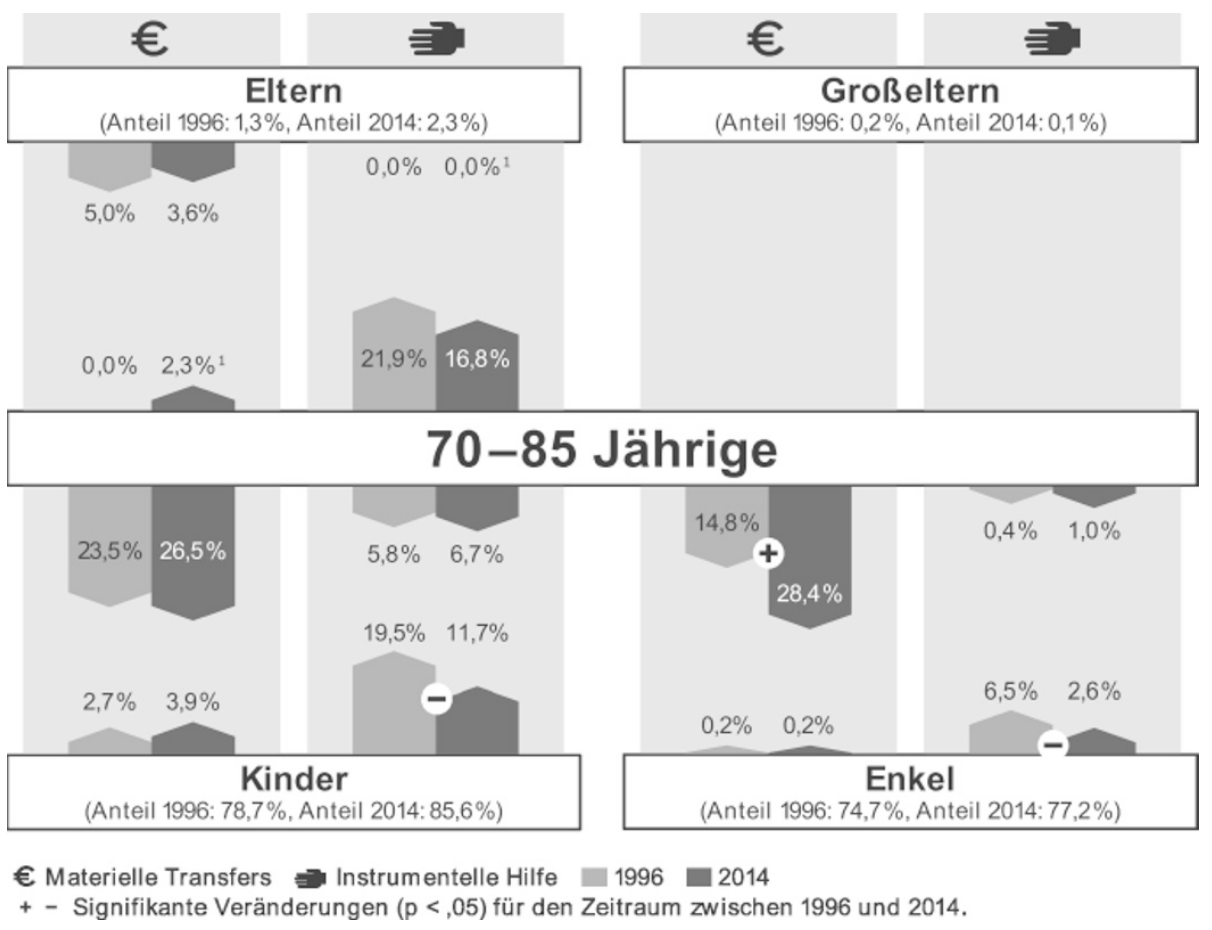

Quelle: DEAS $1996(n=4.838), 2014(n=6.002)$, gewichtet, gerundete Angaben.

1 Aufgrund geringer Fallzahlen konnten keine Tests zur Prüfung signifikanter Unterschiede zwischen 1996 und 2014 durchgeführt werden. Nicht berichtete Transferraten beruhen auf einer Gruppengröße $\mathrm{n} \leq 10$.

\section{Intergenerationale Transfers folgen 2014 demselben Muster wie 1996.}

Angesichts der genannten Verschiebungen kristallisiert sich im Jahr 2014 noch deutlicher als für 1996 das für die Beschreibung des Austausches zwischen den Generationen geprägte Kaskadenmodell heraus: Generationenabwärts fließende Transfers - unabhängig ihrer Art - übersteigen die in entgegengesetzter Richtung erbrachte Unterstützung. Die Bilanz von Nehmen und Geben fällt zugunsten der jüngeren Generationen aus, was am Beispiel der mittleren Altersgruppe verdeutlicht werden soll: Im Jahr 2014 unterstützen von den 55- bis 69-jährigen Eltern mit volljährigen und selbstständigen Kindern 37,5 Prozent ihre Kinder materiell und 13,4 Prozent unterstützen sie instrumentell. Umgekehrt erhalten 2,7 Prozent materielle und 10,5 Prozent instrumentelle Hilfe von ihren Kindern.
Reziprok ist das Transfergeschehen insofern weiterhin, als trotz der unausgeglichenen Gesamtbilanz (die sich offenbar auch nicht über den Verlauf der Familienphasen hinweg ausgleicht) eine Gegenseitigkeit besteht: In fast allen betrachteten Beziehungstypen kann der Tausch von materiellen und instrumentellen Hilfen beobachtet werden. Zwar fließen häufiger materielle Ressourcen von älteren zu jüngeren Generationen, doch umgekehrt fällt die Bilanz instrumenteller Hilfen zugunsten der jeweils älteren Generation aus. Dieses Bild zeigt sich umso stärker, je älter die Generationenbeziehungen sind und spiegelt damit offenbar altersspezifische Bedarfe wider: So unterstützen im Jahr 2014 beispielsweise 26,5 Prozent der 70-bis 85-Jährigen ihre erwachsenen Kinder materiell und 3,9 Prozent erhalten vergleichbare Transfers. Mit Blick auf instrumentelle Hilfe stellt sich das Verhältnis umgekehrt dar: 6,7 Prozent geben entsprechende Hilfe an Kinder und 11,7 
Prozent erhalten sie. Das bedeutet, dass die ältere Generation häufiger als die jüngere Generation Geld- und Sachleistungen gibt, doch zugleich erhält sie häufiger instrumentelle Hilfe.

\section{Die Häufigkeit materieller Transfers von Großeltern an Enkelkinder hat sich zwischen 1996 und 2014 fast verdoppelt.}

Grundsätzlich konzentriert sich der Tausch von Hilfe und Unterstützung auf benachbarte Generationen. So fallen fast durchweg die Transferquoten von und $\mathrm{zu}$ den eigenen Eltern und Kindern deutlich höher aus, als von und $\mathrm{zu}$ den Großeltern und Enkelkindern. Doch bereits 2008 hatte sich angedeutet, dass materielle Transfers von Großeltern zunehmend direkt an Enkelkinder fließen, statt dass sie vermittelt über die Kindergeneration die Enkelkinder erreichen (Mahne \& Motel-Klingebiel, 2010). Dieser Trend setzt sich im Jahr 2014 fort: So haben sich in der mittleren (55- bis 69-Jährige) und der ältesten Gruppe (70- bis 85-Jährige) jeweils die Prävalenzraten materieller Transfers an Enkelkinder zwischen 1996 und 2014 beinahe verdoppelt: Von 8,1 Prozent auf 15,8 Prozent beziehungsweise von 14,8 Prozent auf 28,4 Prozent. $\mathrm{Da}$ insbesondere in den beiden ältesten Altersgruppen (das heißt ab dem 55. Lebensjahr) im Jahr 2014 im Durchschnitt weniger Enkelkinder vorhanden sind als noch im Jahr 1996, fallen diese steigenden Transferraten mit weniger Enkelkindern zusammen. Entsprechend ist $\mathrm{zu}$ vermuten, dass vor allem Enkelkinder von den veränderten Transferflüssen profitieren.

\section{Das Austauschmuster unterscheidet sich nicht zwischen Männern und Frauen.}

Insgesamt zeigen sich keine bedeutsamen Geschlechtsunterschiede. Anders als für die Enkelkinderbetreuung (vgl. Kapitel 15), die Übernahme von Hausarbeit (vgl. Kapitel 13) sowie die Unterstützung, die im Fall gesundheitlicher Einschränkungen für andere erbracht wird (vgl. Kapitel 12), finden sich für den intergenerationalen Austausch von Unterstützung keine Hinweise für die Vermutung, dass Frauen hieran stärker beteiligt wären (vgl. Tabellen A 16-1 bis A 16-4 im Anhang). Sowohl für materielle aber auch instrumentelle Hilfen zeigen sich in den einzelnen Generationenbeziehungen keine systematischen Geschlechtsunterschiede. Auch lassen sich keine nennenswerten geschlechtsspezifischen Veränderungen der Prävalenzraten zwischen den Jahren 1996 und 2014 erkennen. Lediglich die instrumentelle Hilfe, die für Eltern erbracht wird, hat unter den Männern der zweiten Lebenshälfte signifikant an Häufigkeit gewonnen - nicht aber unter den Frauen (vgl. Tabelle A 16-3 im Anhang). Dieser auf Männer beschränkte Anstieg ist möglicherweise Ausdruck dafür, dass die Zeitressourcen der Frauen bereits stark durch die Übernahme anderer familialer Aufgaben wie Hausarbeit und Enkelbetreuung in Verbindung mit wachsender Erwerbsbeteiligung beansprucht sind.

\subsection{Diskussion und Implikationen}

Mit der Vertikalisierung der Familienstruktur, wonach immer häufiger Mitglieder mehrerer Generationen gleichzeitig leben, hat sich der Möglichkeitsspielraum, eventuell aber auch die Notwendigkeit für den Austausch von Geldund Sachleistungen oder Hilfen bei der Alltagsführung zwischen den Generationen vergrößert. Dabei sind intergenerationale Transfers nicht nur Ausdruck des aktuellen Zusammenhalts zwischen den Generationen, sondern stellen zugleich eine Investition in zukünftige Bedarfssituationen dar. So bilden materielle und instrumentelle Hilfen zwischen den Generationen eine tragende Säule der individuellen Daseinsvorsorge und sind gerade in Notfallsituationen ein flexibles und wirkungsvolles Instrument, Risiken des Lebens abzufangen oder zumindest abzumildern. Entsprechend würde ein Rück- 
gang gegenseitiger Transferleistungen innerhalb der Familie einen Verlust bedeuten, vor allem dann, wenn dieser von den Generationen nicht gewollt ist, sondern das Resultat fehlender materieller oder zeitlicher Ressourcen oder ungünstiger Gelegenheitsstrukturen ist. Werden dann benötigte Hilfen und Unterstützung nicht durch andere, professionelle oder institutionalisierte Quellen kompensiert, besteht die Gefahr, dass Versorgungsdefizite entstehen.

Die vorliegenden Auswertungen bieten keine Anhaltspunkte für einen Rückgang intergenerationaler Unterstützung innerhalb der Familie. So ist die Gesamthäufigkeit der Transfers zwischen den familialen Generationen weitgehend stabil geblieben. Personen zwischen 40 und 85 Jahren empfangen im Jahr 2014 nicht nur Hilfe von anderen Familienmitgliedern, sondern sind selbst auch oft Quelle von Unterstützung. In finanzieller Hinsicht deutet sich sogar an, dass sie 2014 stärker noch als 1996 eine wichtige Institution zur Umverteilung materieller Ressourcen sind. Der Befund gestiegener materieller Transferraten an Familienmitglieder ist zudem vor dem Hintergrund weiterer Trends zu bewerten wie die Zunahme der Betreuungsquote von Enkelkindern (vgl. Kapitel 15) und der Anstieg des Anteils derer, die kranke Angehörige unterstützen und pflegen (vgl. Kapitel 12). Aus diesen Befunden kann auf ein außerordentliches Unterstützungspotenzial der heutigen 40 - bis 85-Jährigen geschlossen werden.

Allerdings lässt sich insbesondere der hier beobachtete Anstieg materieller Transferraten an nachfolgende Familiengenerationen auch als eine einsetzende Verschiebung intergenerationaler Abhängigkeitsverhältnisse verstehen: Während sich die (finanzielle) Unabhängigkeit der älteren Generationen weiterhin vergrößert hat - hauptsächlich bedingt durch deren verbesserte Einkommens- und Vermögenslage (vgl.
Kapitel 6 und 7; Vogel \& Künemund 2014) sind die jüngeren Generationen vermutlich stärker auf die finanzielle Unterstützung durch ihre (Groß-)Eltern angewiesen. Eine solche Entwicklung birgt durchaus Risiken in sich: So könnten die Generationenbeziehungen überlastet werden, vor allem wenn gesteigerte Erwartungen von instrumentellen Gegenleistungen, aber auch von emotionaler Zuwendung oder gemeinsam verbrachter Zeit von den jüngeren Familiengenerationen nicht erfüllt werden wollen oder nicht erfüllt werden können. Das dürfte ein gewisses Potenzial für Konflikte und Ambivalenzen innerhalb des familialen Beziehungsgeflechtes mit sich bringen. Darüber hinaus ist eine solche finanzielle Abhängigkeit der jüngeren von älteren Familiengenerationen auch insofern riskant, als dass sich die Wirtschaftslage zukünftiger Eltern- und Großelterngenerationen wieder verschlechtern kann. Ein (Wieder-) Anstieg von Altersarmut wird bereits prognostiziert (Vogel \& Künemund 2014). Damit würde nicht nur eine wichtige Unterstützungsquelle jüngerer Generationen verloren gehen, zugleich könnten dadurch die älteren Generationen wieder selbst verstärkt auf Hilfen ihrer erwachsenen Nachkommen angewiesen sein. Dementsprechend sollten - trotz des offenbar beträchtlichen Potenzials intergenerationaler Solidarität - sozialpolitische Maßnahmen darauf bedacht sein, die gewonnene Selbstständigkeit der Familiengenerationen $\mathrm{zu}$ erhalten beziehungsweise weiter $\mathrm{zu}$ fördern. Das meint nicht nur eine ausreichende Absicherung im Rentenalter, sondern auch die finanzielle und alltagspraktische Unterstützung junger (Familien-)Generationen. An erster Stelle sollte es allerdings darum gehen, die Möglichkeiten einträglicher (Frauen-)Erwerbstätigkeit weiterhin zu verbessern, um auf diese Weise eine selbstständige Lebensführung $\mathrm{zu}$ ermöglichen.

\section{Literatur}

Albertini, M., \& Kohli, M. (2013). The generational contract in the family: An analysis of transfer regimes in Europe. European Sociological Review, 29(4), 828-840.
Alessie, R., Angelini, V., \& Pasini, G. (2014). Is it true love? Altruism versus exchange in time and money transfers. De Economist, 162(2), 193-213. 
Deindl, C., Brandt, M., \& Hank, K. (2014). Generationen in Europa: Theoretische Perspektiven und empirische Befunde. In: A. Steinbach, M. Hennig \& O. Arránz Becker (Hrsg.) Familie im Fokus der Wissenschaft (Familienforschung, S. 299-325). Wiesbaden: Springer.

Hank, K. (2015). Intergenerationale Beziehungen. In: P. B. Hill \& J. Kopp (Hrsg.) Handbuch Familiensoziologie (S. 463-486). Wiesbaden: Springer VS.

Igel, C. (2012). Finanzielle Transfers (Großeltern in Europa). Wiesbaden: VS Verlag für Sozialwissenschaften.

Kahn, J. R., McGill, B. S., \& Bianchi, S. M. (2011). Help to Family and Friends: Are There Gender Differences at Older Ages? Journal of Marriage and Family, 73(1), 7792. doi: 10.1111/j.1741-3737.2010.00790.x.

Kohli, M., \& Künemund, H. (2001). Geben und Nehmen. Die Älteren im Generationenverhältnis. Zeitschrift für Erziehungswissenschaft, 4(4), 513-528.

Kohli, M., Künemund, H., Motel-Klingebiel, A., \& Szydlik, M. (2005). Generationenbeziehungen. In: M. Kohli \& H. Künemund (Hrsg.) Die zweite Lebenshälfte: Gesellschaftliche Lage und Partizipation im Spiegel des Alters-Survey (Bd. 2, S. 157-175). Wiesbaden: VS Verlag für Sozialwissenschaften.

Künemund, H., \& Rein, M. (1999). There is more to receiving than needing: theoretical arguments and empirical explorations of crowding in and crowding out. Ageing \& Society, 19(01), 93-121.

Litwin, H., Vogel, C., Künemund, H., \& Kohli, M. (2008). The balance of intergenerational exchange: correlates of net transfers in Germany and Israel. European journal of ageing, 5(2), 91-102.
Mahne, K., \& Motel-Klingebiel, A. (2010). Familiale Generationenbeziehungen. In: A. Motel-Klingebiel, S. Wurm \& C. Tesch-Römer (Hrsg.) Altern im Wandel. Befunde des Deutschen Alterssurveys (DEAS) (S. 188214). Stuttgart: Kohlhammer.

Marbach, J. H. (1997). Sozialer Tausch unter drei familiär verbundenen Generationen. In: J. Mansel (Hrsg.) Generationen-Beziehungen, Austausch und Tradierung (S. 85-96). Opladen: Westdeutscher Verlag.

Motel-Klingebiel, A., Mahne, K., \& Huxhold, O. (2010). Was treibt Transfers zwischen Eltern und erwachsenen Kindern an? Zur Dynamik familialer Generationenbeziehungen im späten Lebenslauf. In: A. Ette, K. Ruckdeschel \& R. Unger (Hrsg.) Potenziale intergenerationaler Beziehungen: Chancen und Herausforderungen für die Gestaltung des demografischen Wandels. Würzburg: Ergon Verlag.

Motel-Klingebiel, A., Tesch-Römer, C., \& Kondratowitz, H.-J. von (2005). Welfare states do not crowd out the family: evidence for mixed responsibility from comparative analyses. Ageing and society, 25(06), 863-882.

Rosenthal, C. J. (1985). Kinkeeping in the familial division of labor. Journal of Marriage and the Family, 47(4), 965-974.

Vogel, C. (2008). Generationenbilanzen familialer Unterstützungsleistungen im europäischen Vergleich. Vechta: Zentrum Altern und Gesellschaft der Hochschule Vechta.

Vogel, C., \& Künemund, H. (2014). Armut im Alter. In: S. Frech \& O. Groh-Samberg (Hrsg.) Armut in Wohlstandsgesellschaften (S. 123-136). Schwalbach im Taunus: Wochenschau Verlag.

Open Access Dieses Kapitel wird unter der Creative Commons Namensnennung 2.5 International Lizenz (http://creativecommons.org/licenses/by/2.5/deed.de) veröffentlicht, welche die Nutzung, Vervielfältigung, Bearbeitung, Verbreitung und Wiedergabe in jeglichem Medium und Format erlaubt, sofern Sie den/die ursprünglichen Autor(en) und die Quelle ordnungsgemäß nennen, einen Link zur Creative Commons Lizenz beifügen und angeben, ob Änderungen vorgenommen wurden.

Die in diesem Kapitel enthaltenen Bilder und sonstiges Drittmaterial unterliegen ebenfalls der genannten Creative Commons Lizenz, sofern sich aus der Abbildungslegende nichts anderes ergibt. Sofern das betreffende Material nicht unter der genannten Creative Commons Lizenz steht und die betreffende Handlung nicht nach gesetzlichen Vorschriften erlaubt ist, ist für die oben aufgeführten Weiterverwendungen des Materials die Einwilligung des jeweiligen Rechteinhabers einzuholen. 